\title{
Effect of geometry modifications on the engulfment in micromixers: numerical simulations and stability analysis
}

\author{
L. Siconolfi ${ }^{\mathrm{a}}$, A. Fani ${ }^{\mathrm{b}}$, S. Camarri ${ }^{\mathrm{a}}$, M.V. Salvetti ${ }^{\mathrm{a}, *}$ \\ ${ }^{a}$ Dipartimento di Ingegneria Civile e Industriale, Università di Pisa, \\ Via G. Caruso 8, 56122 Pisa, Italy \\ ${ }^{b}$ Laboratory of Fluid Mechanics and Instabilities, Ecole Polytechnique Fédérale de \\ Lausanne, Lausanne, Switzerland
}

\begin{abstract}
The effect of the geometry variations on the engulfment regime in micromixers is investigated. In particular, starting from a T-mixer, the complementary angle $\alpha$ between the axes of the inlet channels and that of the outflow conduit is varied between $\alpha=-20^{\circ}$ (arrow-like mixers) to $\alpha=+5^{\circ}$ (Y-mixers), $\alpha=0^{\circ}$ denoting a T-mixer. It is shown by direct numerical simulations (DNS) that the engulfment regime is present in all the considered geometries and it starts at progressively lower values of the flow Reynolds number as $\alpha$ is decreased. The main differences in the flow topology are limited and mainly confined at the confluence region between the two incoming flows. The instability leading to the engulfment regime is further investigated by linear stability analysis. This allows a more accurate prediction of the critical Reynolds number for the onset of engulfment as $\alpha$ is varied and, through
\end{abstract}

\footnotetext{
${ }^{*}$ Corresponding author

Email addresses: siconolfi.lorenzo@gmail.com (L. Siconolfi), andrea.fani@epfl.ch (A. Fani), s.camarri@ing.unipi.it (S. Camarri), mv.salvetti@ing.unipi.it (M.V. Salvetti)
} 
an adjoint-based sensitivity analysis, the localization of the corresponding instability core.

Keywords: micromixers, engulfment, flow instability, geometry variations

\section{Introduction}

Fast and efficient mixing at the microscale is important in the process industry (e.g. miniature fuel cells or micro-reactors) as well as in the biological field (e.g. molecular diagnostics). Passive micromixers, where mixing is promoted without the help of any ad-hoc external power source [1], are particularly interesting because they are inexpensive and simple to operate. One of the simplest designs of passive micromixers is a $\mathrm{T}$ shape, in which the inlet channels join the main mixing channel with T-shaped branches. This type of mixers is often encountered as a junction element in more complex micro-systems. The flow behavior and the mixing efficiency of T-mixers have been largely investigated in the literature. The following flow regimes have been identified, depending on the Reynolds number, Re: the stratified, the vortex, the engulfment and the unsteady flow regimes (see e.g. [2-9]). In the stratified flow regime, the inlet streams come in contact with one another in the mixing zone and then flow side by side through the mixing channel, resulting in a completely segregated fluid flow. In the vortex regime, above a critical $R e$, a secondary flow in the form of a double vortex pair occurs, leaving however the two incoming streams well segregated. As Re is increased, the flow field goes through a bifurcation which drives the system from a per-

fectly symmetric flow to an asymmetric configuration, generally referred to as the engulfment regime. Here, convection plays a major role in the mixing, 
which is, hence, largely increased. Finally, by further increasing $R e$, the flow becomes first unsteady and periodic in time and eventually chaotic [5-7, 9].

However, the engulfment regime is so far the most studied one, due to its high mixing efficiency (e.g. $[4,3,10-13,8,14,15])$. As for the flow characterization in the engulfment regime, it was shown in [8], through direct numerical simulations (DNS) and global stability analysis, that a key feature of the engulfment regime is the tilting of the three-dimensional vortical structures forming at the confluence of the two fluid streams. This causes the break of symmetry of the vorticity field in the mixing channel, which is characterized, then, by the presence of two dominant co-rotating vortices, yielding to the increase of mixing. It was also found that the core of the instability, i.e. the region where the flow instability leading to the engulfment onset originates, coincides with the recirculation regions near the top wall at the confluence between the two incoming streams, where the previous threedimensional vortices form. This is in agreement with the proposed scenario for the engulfment onset.

Another practical issue, which received a large attention in the literature, is how different geometrical and flow parameters, besides the Reynolds number, affects the engulfment onset and characteristics. For instance, the effect of the velocity profile of the incoming streams was investigated in [13] and in [8], showing that a fully-developed "Poiseuille-like" velocity profile tends to promote the engulfment onset, compared to a non fully-developed flatter profile. As for the geometry, important parameters are the aspect ratios of the inlet and outlet channels. The aspect ratio of the outlet mixing channel is usually chosen equal to twice that of the inlet channels, in order to 
have the same bulk velocity in the mixing channel as in the inlet ones. Although the most studied T-mixer configuration is the one with square inlet channels, the effects of varying the inlet aspect ratio have been investigated in $[10,11,14,15]$, showing that this parameter may have a significant impact on the engulfment onset. More sophisticated and complex geometry modifications have also been proposed (see, e.g., [7]).

In the present paper, we focus on geometrical modifications of a T-mixer configuration obtained by varying the angle between the axis of the inlet channels and that of the mixing conduit (in the T-mixer these axes are perpendicular); this leads to "arrow-like" or Y mixer configurations. The channel aspect ratios and the other geometrical parameters are kept unchanged. Limited variations of the angle are considered, so that the new geometries may be considered as perturbations of the reference T-mixer configuration; in any cases the modified geometries are very simple to be obtained in practice. Nonetheless, such geometry modifications may be expected to have a significant impact on the engulfment onset and patterns, since they operate in the region where the engulfment instability originates and where the vortical structures characterizing this regime form. In particular, we wish to investigate (i) whether the considered geometry modifications can promote or delay the engulfment onset and (ii) whether the key physical mechanisms leading to the engulfment, as well the flow patterns, remain the same or not. To this aim we use the same methodology as in [8]. The effects of the angle variation on the engulfment onset and on the physical mechanisms leading to the engulfment regime are first investigated and quantified by DNS. Fully three-dimensional global stability and sensitivity analyses complement the 
DNS study, providing a more accurate estimation of the critical Reynolds number for the engulfment onset, as well as the identification of the instability nature and the spatial distribution of the unstable mode and of the instability core.

\section{Geometry definition and methodology}

The considered micromixer geometries consist of two inlet and one outlet channels, all with rectangular cross sections (see Fig. 1 for a sketch). Different geometries are considered, by varying the angle $\alpha$ between the axis of the inlet channels and the $x$ axis (see Fig. 1). For positive values of $\alpha$ a $\mathrm{Y}$ geometry is obtained, while for negative $\alpha$ the mixer geometry becomes "arrow-like". In all cases, the aspect ratio of inlet channels, $W / h$, is equal to $0.75, h$ being the mixer height and the aspect ratio of the outlet mixing channel is twice that of the inlet channels, i.e. $W_{o} / h=1.5$. Thus, the bulk velocity, $U_{b}$, is the same in the inlet branches as in the outlet conduit. All the lengths are herein normalized by using the hydraulic diameter of the outlet channel $D$. With this normalization, $W=0.625, W_{o}=1.25$ and $h \simeq 0.83$. The length of the inlet channels is $L_{i n}=6.875$, while the one of the mixing

channel $L_{\text {out }}$, is long enough to ensure that the flow field and the complex vortical structures are completely described. More specifically, $L_{\text {out }}=25$ is used in all the simulations.

The motion of an incompressible fluid with constant viscosity, $\mu$, and density, $\rho$ is considered. Thus the governing equations are the unsteady incompressible Navier-Stokes equations, written here in a non dimensional 
form, by using $D$ and $U_{b}$ as reference length and velocity:

$$
\frac{\partial \mathbf{U}}{\partial t}+\mathbf{U} \cdot \nabla \mathbf{U}+\nabla P=\frac{1}{R e} \nabla^{2} \mathbf{U} ; \quad \nabla \cdot \mathbf{U}=0
$$

where $\mathbf{U}=(U, V, W)$ is the fluid velocity, $P$ the reduced pressure and $R e=U_{b} D / \nu$ is the Reynolds number, with $\nu=\mu / \rho$ denoting the kinematic viscosity.

No-slip boundary conditions are imposed at the mixer walls, while, at the inlet, a unidirectional fully developed velocity profile is assumed. The latter can be derived by solving the steady-state Navier-Stokes equations with no-slip boundary conditions at the walls and subjected to a constant axial pressure gradient (see $[16,17,15]$ for more details). Finally, with reference to Fig. 1, free outflow conditions of the following type are used: $\partial_{y} U=$ $0, P-R e^{-1} \partial_{y} V=0 \partial_{y} W=0$.

The instability onset is studied using linear theory and normal-mode analysis, in which the flow variables are decomposed in the base flow $\left(\mathbf{U}_{b}, P_{b}\right)$, steady solution of equations (1), and in an unsteady perturbation $(\mathbf{u}, p)$. The perturbation is searched in the form of normal modes $\mathbf{q}(x, y, z, t)=$ $(\mathbf{u}, p)(x, y, z, t)=(\hat{\mathbf{u}}, \hat{p})(x, y, z) \exp (\sigma t)$, where $\sigma$ is a generally complex eigenvalue associated with the mode $(\hat{\mathbf{u}}, \hat{p})$. Introducing the flow decomposition and the normal-mode form into Eqs. (1) and linearizing in the disturbance amplitude, we obtain the following equations governing the dynamics of the perturbation:

$$
\begin{aligned}
& \sigma \hat{\mathbf{u}}+\hat{\mathbf{u}} \cdot \nabla \mathbf{U}_{b}+\mathbf{U}_{\mathbf{b}} \cdot \nabla \hat{\mathbf{u}}+\nabla \hat{p}-\frac{1}{R e} \nabla^{2} \hat{\mathbf{u}}=\mathbf{0} \\
& \nabla \cdot \hat{\mathbf{u}}=0
\end{aligned}
$$

The boundary conditions associated with Eq. (2) are: $\hat{\mathbf{u}}=0$ at the inlet 
surfaces and on the walls and free outflow conditions at the outlet. Equations (2) along with the boundary conditions are an eigenvalue problem. The flow is linearly stable when all the eigenvalues, $\sigma=\lambda+\imath \omega$, are characterized by $\lambda<0$.

In order to identify the core of the instability [21], the mode adjoint to $(\hat{\mathbf{u}}, \hat{p}, \sigma)$ is also computed as the solution of the following eigenvalue problem:

$$
\begin{aligned}
& \sigma^{*} \hat{\mathbf{u}}^{+}+\nabla \mathbf{U}_{b} \cdot \hat{\mathbf{u}}^{+}-\mathbf{U}_{\mathbf{b}} \cdot \nabla \hat{\mathbf{u}}^{+}+\nabla \hat{p}^{+}-\frac{1}{R e} \nabla^{2} \hat{\mathbf{u}}^{+}=\mathbf{0} \\
& \nabla \cdot \hat{\mathbf{u}}^{+}=0
\end{aligned}
$$

The boundary conditions associated with problem (3) are: $\hat{\mathbf{u}}^{+}=0$ at the inlet surfaces and on the lateral walls, and $p^{+} \mathbf{n}-R e^{-1}\left(\mathbf{n} \cdot \nabla \hat{\mathbf{u}}^{+}\right)=\left(\mathbf{U}_{\mathbf{b}} \cdot \mathbf{n}\right) \hat{\mathbf{u}}^{+}$ at the outlet (see [18]). The adjoint velocity field is normalized as follows $\left(\hat{\mathbf{u}}^{+}, \hat{\mathbf{u}}\right)=1$, where $(\cdot, \cdot)$ is the $L_{2}$ scalar product in the computational domain.

Direct numerical simulations are carried out by discretizing Eq. through the Nek5000 code, a massively parallel open source solver for the incompressible Navier-Stokes equation, based on a high-order spectral element method [19]. The basis functions inside each element consist of Legendre polynomials of $N$-th order for velocity, and $(N-2)$-th order for pressure, with typically $N \geq 6$. These polynomials are the Lagrangian interpolants based on tensor-product arrays of the Gauss-Lobatto-Legendre (GLL) quadrature points in each hexahedral element. It can be shown that the method has both a spectral convergence in $N$ (i.e., the so-called $p$-refinement), and the convergence of a high order finite element method in the number of elements (i.e., the so-called $h$-refinement). Time discretization uses a third order explicit backward-differentiation of the convective terms, and an implicit scheme for the viscous terms. 
The eigenvalue problems (2) and (3) have been solved by an Arnoldi iteration method ad-hoc implemented by the authors in NEK5000. The linearized/adjoint version of the code has been used as a time-stepper. The base flow is obtained as the asymptotic steady solution of the non linear DNS simulations.

A structured multi-block grid was used for all the simulations. Along the width of all the channels, a uniform element size of 0.07 is chosen, while along their axis the size varies between 0.08 and 0.5 . In the $z$ direction, the element size is uniform equal to 0.083. The polynomial order for the velocity is equal to 6. Globally, the used grid has 15680 elements, associated to approximately $10^{7}$ degrees of freedom for the velocity. Grid convergence of the DNS results has been checked. As concerns the time discretization, a constant time step equal to $7 \times 10^{-4}$ is adopted for both the non-linear simulations and the linearized equations used in the time-stepper approach, leading to a CFL number approximately equal to 0.25 .

\section{Flow behavior for varying geometry}

Direct numerical simulations have been carried out for the following values of $\alpha$ : $-20^{\circ},-10^{\circ},-5^{\circ},-2^{\circ}, 2^{o}$ and $5^{\circ}$. For each value of $\alpha$, simulations at increasing Reynolds numbers are performed. Figure 2 shows the values of $R e$ at which the flow is found to be in the engulfment regime as a function of $\alpha$. The critical Reynolds number has not been determined precisely from DNS, since simulations were carried for increments of $R e$ equal to 10. Therefore, there is an uncertainty region (the dark grey band in Fig. 2), while more accurate estimations (the white circles) could be obtained by linear stability 


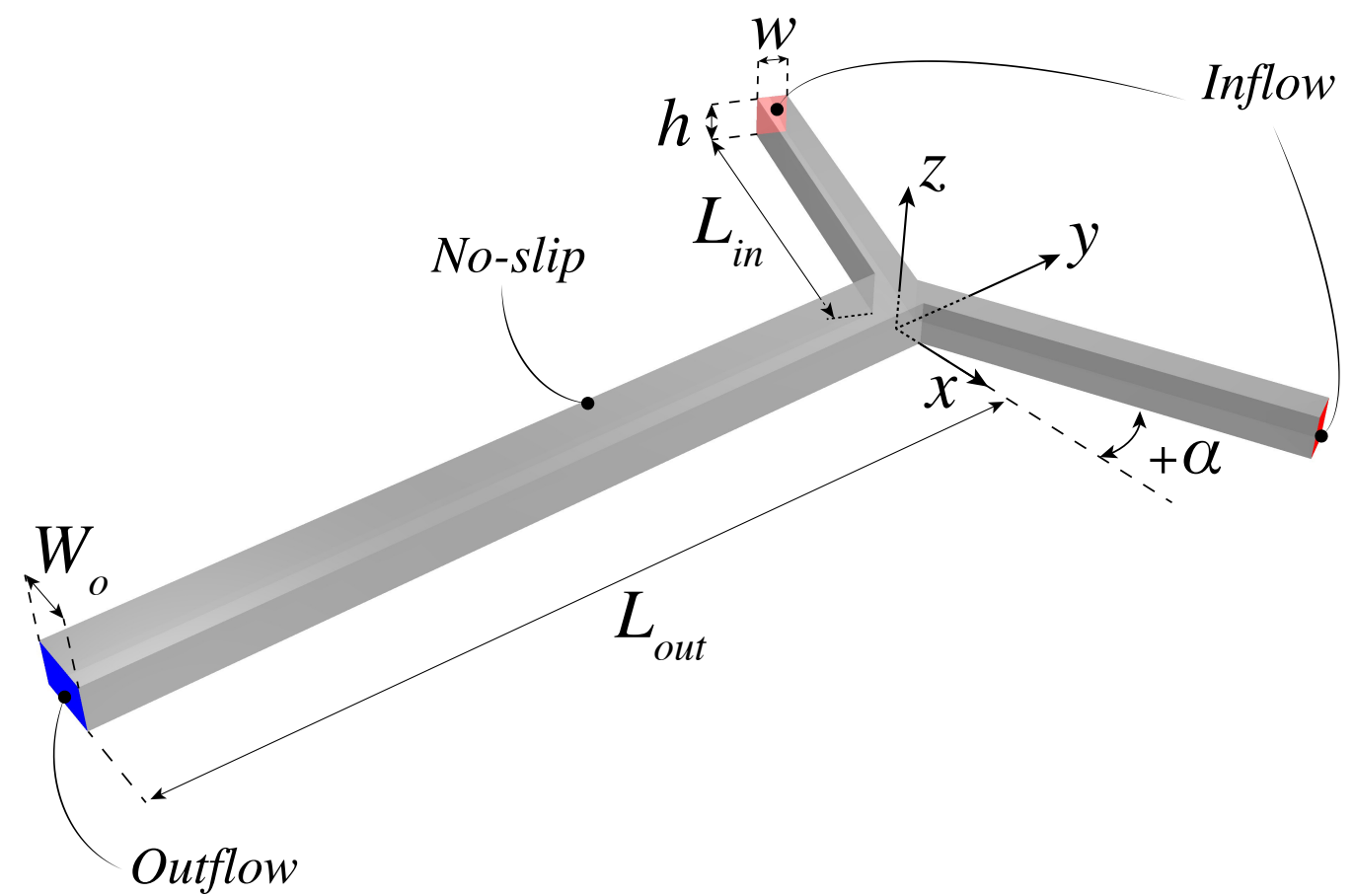

Figure 1: Sketch of the micromixer geometry (not in scale). 


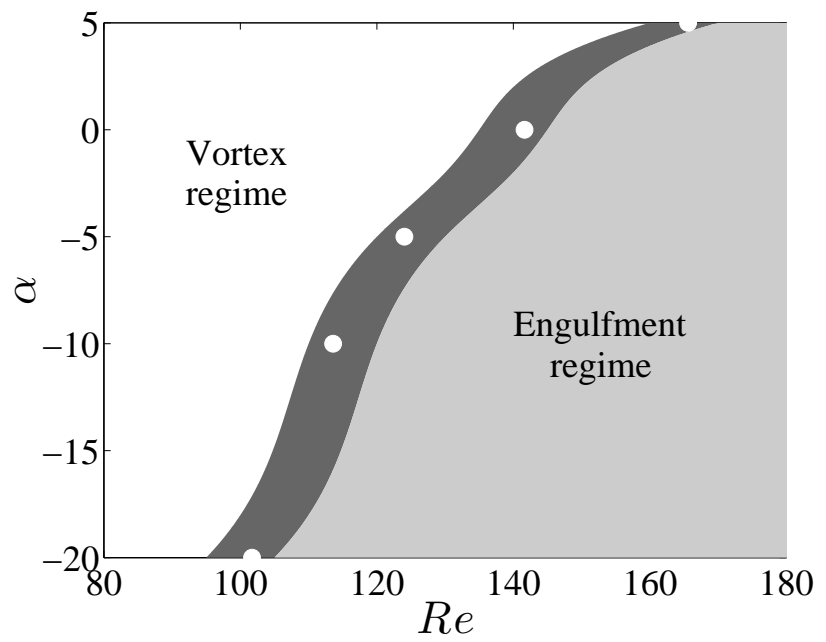

Figure 2: Critical Reynolds number for the engulfment onset estimated from DNS (filled regions) and by the stability analysis (white circles).

analysis, as explained in Sec. 4. It is evident how negatives $\alpha$ angles, and hence arrow-like shapes, promote the onset of the engulfment regime. Conversely, for $\mathrm{Y}$ shapes the engulfment is delayed. This observation led us to investigate a larger range of negative $\alpha$ values.

As for the physical features characterizing the different regimes, Figs. 3(a) and 3(b) show the three-dimensional vortical structures for the mixer configuration with $\alpha=-20^{\circ}$ at $R e=100$, before the engulfment onset, and at $R e=140$, in the engulfment regime, identified by means of the $\lambda_{2}$ criterion [20]. For both $R e$, the flow is characterized by two counter-rotating $3 \mathrm{D}$ vortical structures. The top parts of these structures originate from the recirculation regions near the top and lateral walls of the mixer at the confluence of the two incoming streams. Due to the convection by the flow streams, each structure is also characterized by two legs which enter in the mixing channel, as can be seen also from the isocontours of vorticity plotted, 
together with the in-plane velocity vectors, at two different sections in the mixing channel and reported in Fig. 3. In the pre-engulfment vortical regime, the top parts of the 3D vortical structures are almost perfectly aligned with the $z$ axis and the legs of each structure are equal each other, except for the opposite sign, and also equal to those of the other structure (see Fig. 3(a)). Moving downstream towards the outlet, they remain almost parallel to the $Y$ axis, simply decreasing their intensity because of diffusion and of the interaction with the vorticity of opposite sign generated at the mixer walls. Therefore, the flow in the mixing channel is characterized by two reflectional symmetries respect to the $z=H / 2$ and $x=0$ planes and the two fluid streams remain segregated. On the other hand, in the engulfment regime the top parts of the 3D vortices are tilted (see the top view in Fig. 3(b)). As a consequence, the two legs of each structure are no more equal, one being more intense than the other and this causes the loss of the reflectional symmetries in the mixing channel. In particular, there are two dominant co-rotating vortices inducing a velocity field, which strongly promotes the mixing between the two streams. This scenario is qualitatively the same as the one observed for $\alpha=0$ [8] and figure 4 shows that the same features characterize the engulfment regime also for Y mixers, although it occurs at higher Reynolds numbers, as previously highlighted.

The main differences in the flow fields obtained for varying $\alpha$ concern the recirculation regions near the top walls at the confluence of the two incoming streams, from which the top parts of the 3D vortical structures originate. These recirculations can be seen for instance from the streamlines of the in-plane velocity on a section at $z=H / 2$ for $\alpha=5$ and $-20^{\circ}$, reported 


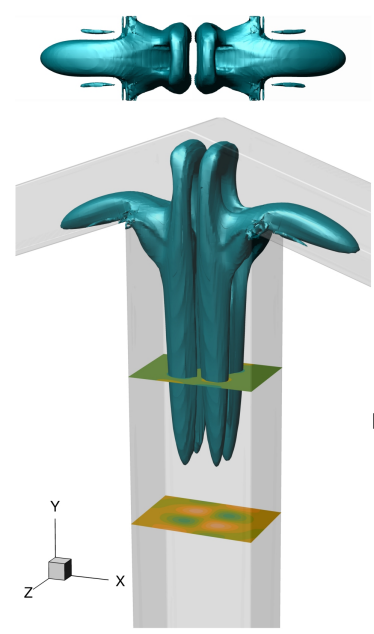

(a)
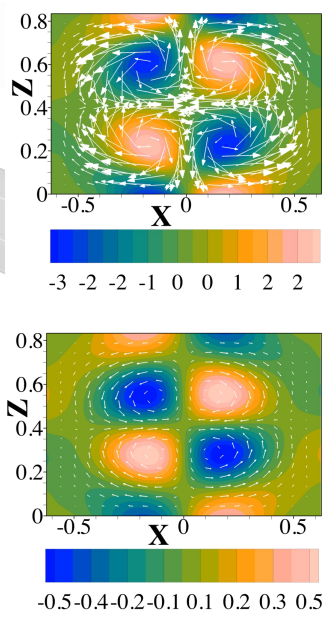

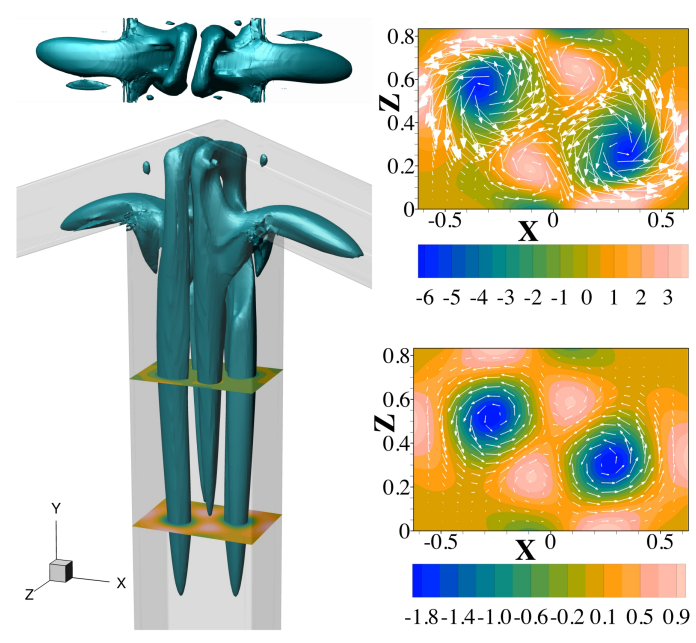

(b)

Figure 3: In each panel, left figures show the 3D vortical structures identified by the $\lambda_{2}$ criterion and right figures show the in-plane velocity vectors and the normal $(y)$ vorticity (sections at $y=-1.5$ at top and $y=-3$ at bottom) for the micromixer having $\alpha=-20^{\circ}$. (a) $R e=100$ vortical regime and (b) $R e=140$ engulfment regime. 


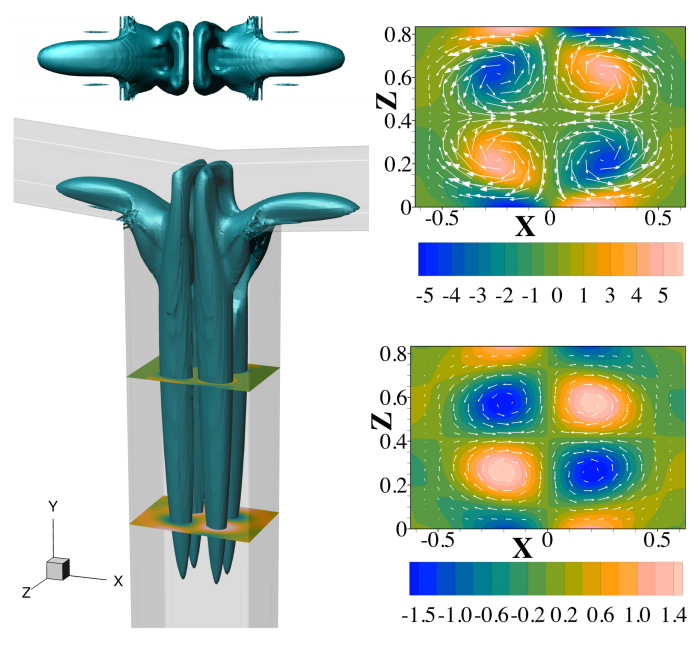

(a)
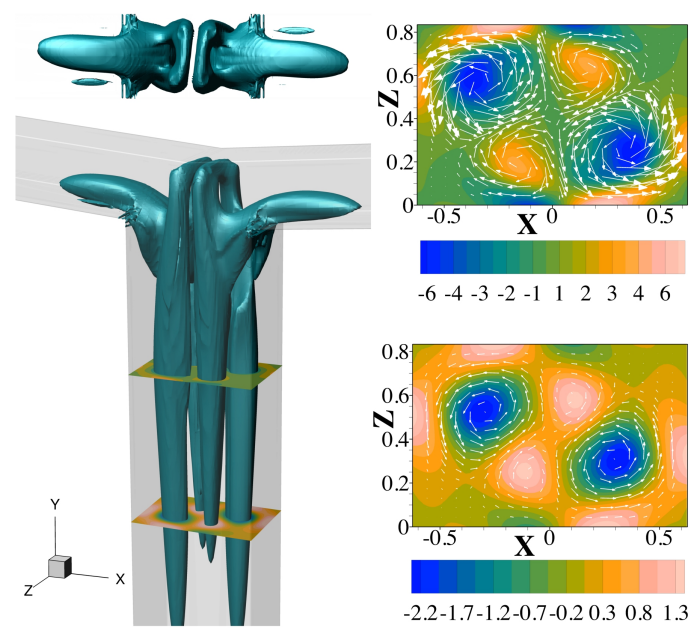

$\begin{array}{llllllllll}-6 & -4 & -3 & -2 & -1 & 1 & 2 & 3 & 4 & 6\end{array}$

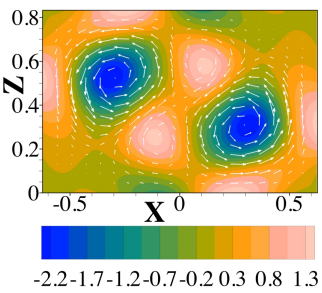

(b)

Figure 4: In each panel, left figures show the 3D vortical structures identified by the $\lambda_{2}$ criterion and right figures show the in-plane velocity vectors and the normal $(y)$ vorticity (sections at $y=-1.5$ at top and $y=-3$ at bottom) for the micromixer having $\alpha=5^{\circ}$. (a) $R e=160$ vortical regime and (b) $R e=170$ engulfment regime. 
in Figs. 5(a) and (b) respectively, at two Reynolds numbers just before the engulfment onset. Isocontours of the module of the in-plane velocity are also reported, while the thick black lines indicate the trace of the 3D vortical structures. It is clear that the recirculation regions in the arrow-like configurations are much larger than in the Y-mixer. Furthermore, in the Ymixer the two recirculations, and consequently the top parts of the vortical structures, form right at the corner at the intersection of the top walls. This geometry constraint probably acts against the tilting of the vortices top parts which characterizes, as previously seen, the engulfment regime. Conversely, for the arrow like configuration the corner at the intersection of the top walls is well inside the recirculation regions; this, together with the larger size of the recirculations, may explain the earlier transition to the engulfment regime occurring for negative $\alpha$ values compared to positive ones. Indeed, as shown in Sec. 4, although the previous outlined differences are very localized, they occur in the region where the instability leading to the engulfment onset originates.

\section{Stability and sensitivity analysis}

Linear stability analysis has been carried out in order to characterize the instability leading to the engulfment regime and its dependence on the mixer geometry, namely on the angle $\alpha$. For each geometry, the analysis is applied to the steady symmetric flow fields obtained by DNS at sub-critical Reynolds numbers. In particular, for each case except for $\alpha= \pm 2^{\circ}$, the stability analysis has been carried out for two different values of $R e, R e_{1}$ and $R e_{2}<R e_{1}$. Thus, an estimation of the critical Reynolds number $R e_{c}$, 

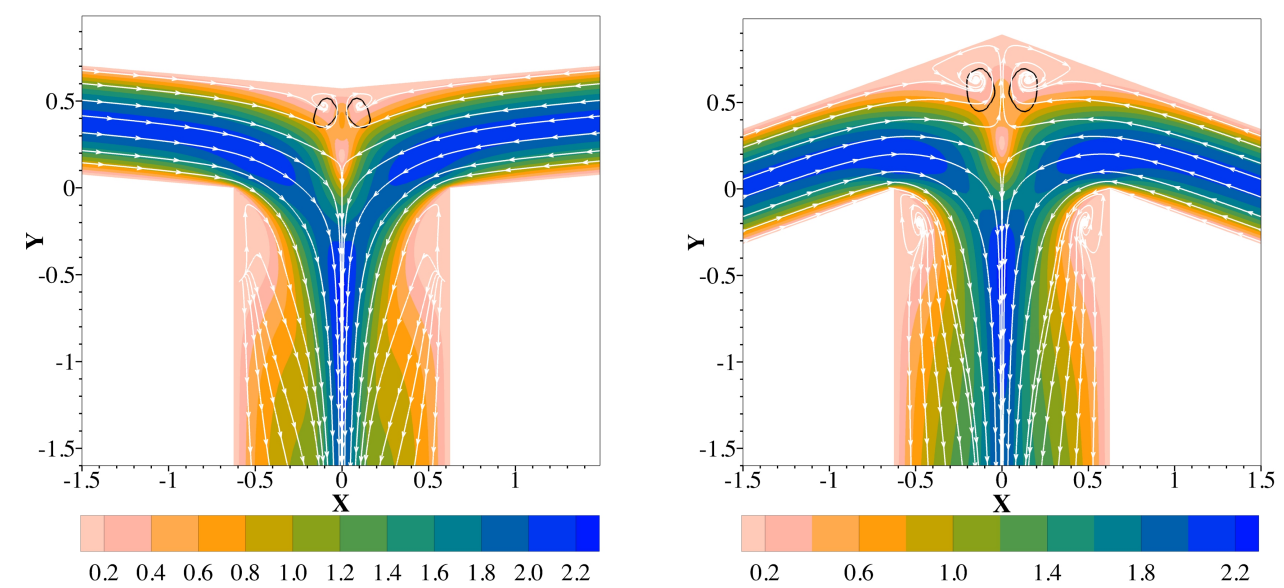

Figure 5: Module and streamlines of the in-plane velocity on the mixer section at $z=H / 2$; the black thick lines indicate the flow region identified as a vortex according to the $\lambda_{2}$ criterion. (a) $\alpha=5^{\circ}$ and $R e=160$; (b) $\alpha=-20^{\circ}$ and $R e=100$

corresponding to a null value of the amplification factor $\lambda$, is obtained by linearly extrapolating $\lambda$ in terms of $R e$ using the values $\lambda_{1}$ and $\lambda_{2}$ obtained for $R e_{1}$ and $R e_{2}$, respectively. As a result, $R e_{c} \simeq R e_{2}-\lambda_{2}\left(R e_{1}-R e_{2}\right) /\left(\lambda_{1}-\right.$ $\left.\lambda_{2}\right)$. For each geometry, $R e_{1}$ has been chosen on the left line identifying the boundary between the vortex and the engulfment regimes in Fig. 2, and we fixed $R e_{2}=R e_{1}-5$. The so-estimated values of $R e_{c}$ are reported in Fig. 2 with white symbols, overlapped to the results obtained by DNS, which, as already commented in Sec. 3, are characterized by an uncertainty band in which the critical Reynolds number for the engulfment onset may lie. The values obtained by linear stability analysis, as described above, lie perfectly inside the DNS uncertainty interval.

As concerns the nature of the identified instability, DNS simulations and the stability analyses indicate that it is a supercritical symmetry-breaking pitchfork bifurcation. Thus, the unstable eigenvalue $\sigma$ is real-valued $(\sigma=\lambda)$, 


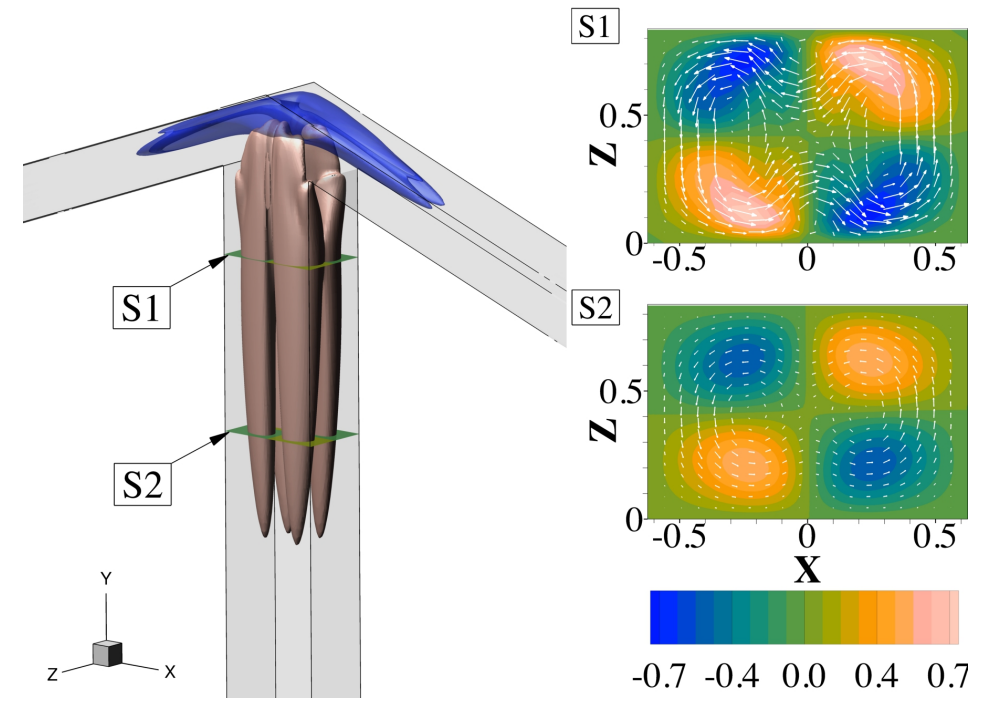

Figure 6: Representative isocosurfaces of the velocity modululs of the direct (light colour) and adjoint (dark colour) eigenmodes for $\alpha=-20^{\circ}$ at $R e=100$ and, on slices S1 and S2, inplane velocity vectors and wall normal velocity (coloured map) of the direct eigenmode.

so as the associated direct $(\hat{\mathbf{u}}, \hat{p})$ and adjoint $\left(\hat{\mathbf{u}}^{+}, \hat{p}^{+}\right)$eigenmodes. As an example of eigenmodes for the case with $\alpha=-20^{\circ}$, we report in Fig. 6 a representative isosurface of the norm of the velocity field of both the direct and adjoint eigenmodes, together with the velocity field of the direct mode at two representative $y$-sections in the outflow channel. Sections S1 and S2 in Fig. 6 show that the velocity field associated with the direct most-unstable mode is a symmetry-breaking mode and, when this is properly weighted and summed to the symmetric base flow used for the stability analysis, the resulting flow field is very similar to that of the engulfment regime reported in Fig. 3 thus proving that the mode is responsible for the onset of the engulfment regime. This is not shown here for the sake of brevity, but we refer to Ref. [8] where this kind of analysis is detailed for a T-mixer. Indeed, 

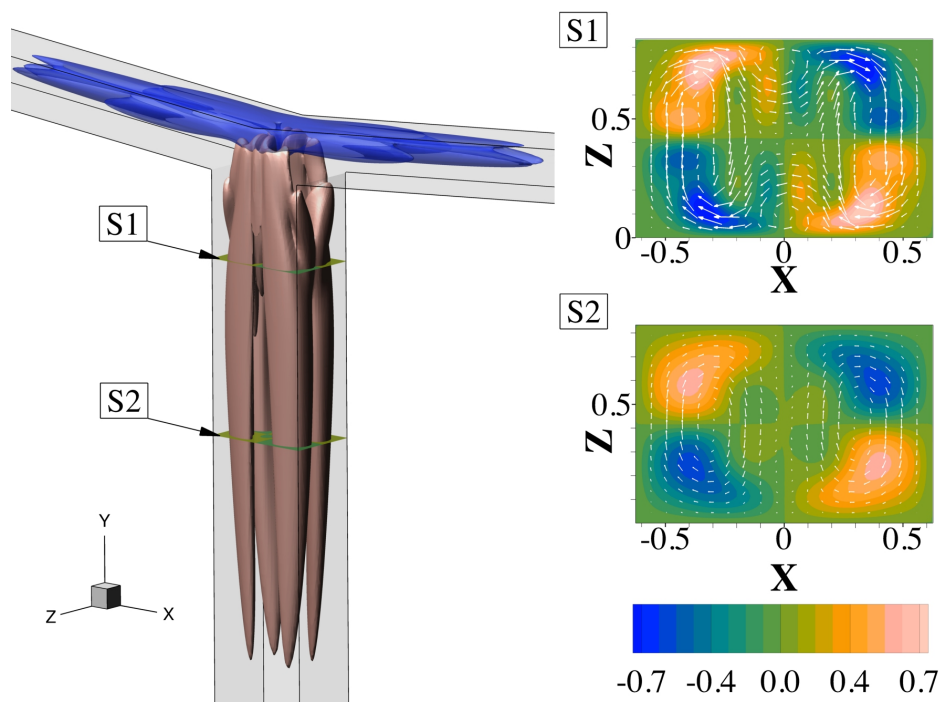

Figure 7: Representative isocosurfaces of the velocity modululs of the direct (light colour) and adjoint (dark colour) eigenmodes for $\alpha=+5^{\circ}$ at $R e=160$ and, on slices S1 and S2, inplane velocity vectors and wall normal velocity (coloured map) of the direct eigenmode.

both the base flow and the identified mode is very similar to that obtained for a T-mixer $\left(\alpha=0^{\circ}\right)$. This fact demonstrates that the nature of the engulfment instability is not altered by the variation of the geometry. The same conclusion can be drawn by the analysis of the case with $\alpha=+5^{\circ}$, whose results are reported in Fig. 7. Indeed, comparing Fig. 6 with Fig. 7 it can be noticed that differences between the engulfment modes for $\alpha=-20^{\circ}$ and $\alpha=+5^{\circ}$ are limited, as it was discussed for the DNS fields in Sec. 3. The main differences are confined at the confluence region between the channels of the mixer, and in particular they concern the position of the modes in the $y$-direction in that region. The differences are directly related to the different shape and position of the recirculation regions of the flow.

As for the case of the T-mixers, Fig. 6 and Fig. 7 show that the direct 
mode originates at the confluence between the two inlet streams and it is convected through the outflow channel. The adjoint mode originates in the same region but, at difference with the direct mode, is convected in the opposite direction (see Eqs. (3)) and occupies mainly the inlet channels. Consequently, the region of overlapping between the direct and the adjoint mode is confined at the confluence of the two inlet channels, where the flow recirculations shown and described in Sec.. 3 are also located. As shown in Ref. [21], the overlapping region between the direct and the adjoint modes identifies the core of the instability. In particular, if a small-amplitude linear force-velocity feedback term is added to the linear stability Eqs. (2), i.e. $\mathbf{f}\left(\mathbf{x}_{0}\right)=\epsilon \mathbf{M} \hat{\mathbf{u}} \delta\left(\mathbf{x}-\mathbf{x}_{\mathbf{0}}\right), \mathbf{M}$ being a generic feedback matrix and $\delta(\mathbf{x})$ the Dirac distributional function, the effect $\delta \sigma$ on the eigenvalue $\sigma$ at first order in $\epsilon$ is given by:

$$
\delta \sigma \simeq \epsilon\left(\hat{\mathbf{u}}^{+}, \mathbf{f}\right) \leq \epsilon\|M\|\left\|\hat{\mathbf{u}}^{+}\right\|\|\hat{\mathbf{u}}\|
$$

where $\|\cdot\|$ indicates a generic vector and the induced matrix norm. In Ref. [21] it is discussed in detail why a perturbation of the considered type is appropriate to identify the regions where the instability takes origin. In the present flows the sensitivity index $S(\mathbf{x})=\left\|\hat{\mathbf{u}}^{+}(\mathbf{x})\right\|\|\hat{\mathbf{u}}(\mathbf{x})\|$ is localized in space due to the peculiar shape of the direct and adjoint eigenmodes and, for this reason, we can identify that region as the core of the instability. An isosurface of the scalar field $S(\mathbf{x})$ is plotted in Fig. 8 and Fig. 9 for the cases with $\alpha=-20^{\circ}$ and $\alpha=+5^{\circ}$, respectively, and the value of the isosurface is such that we can consider $S$ negligibly small externally to the isosurface. Thus, the surface plotted in Figs. 8 and 9 represent the boundary of the instability core. By a comparison of the two figures it is possible to evince that the 

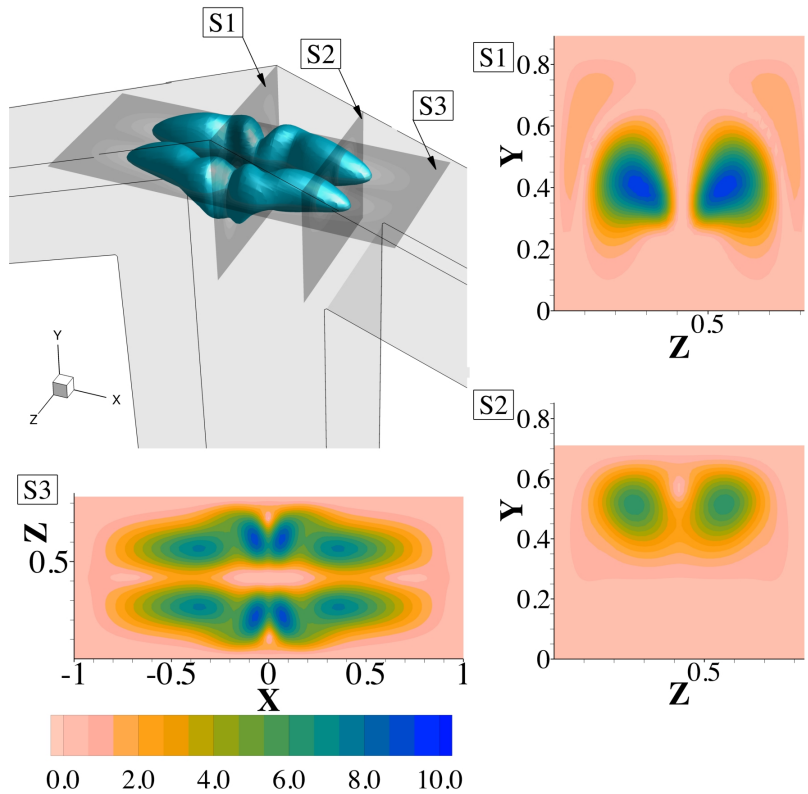

Figure 8: Isosurface of the scalar sensitivity field $S$ containing the core of the instability and distribution of $S$ at the three sections S1, S2 and S3 highlighted in the 3D figure for the case at $\alpha=-20^{\circ}$ and $R e=100$. 

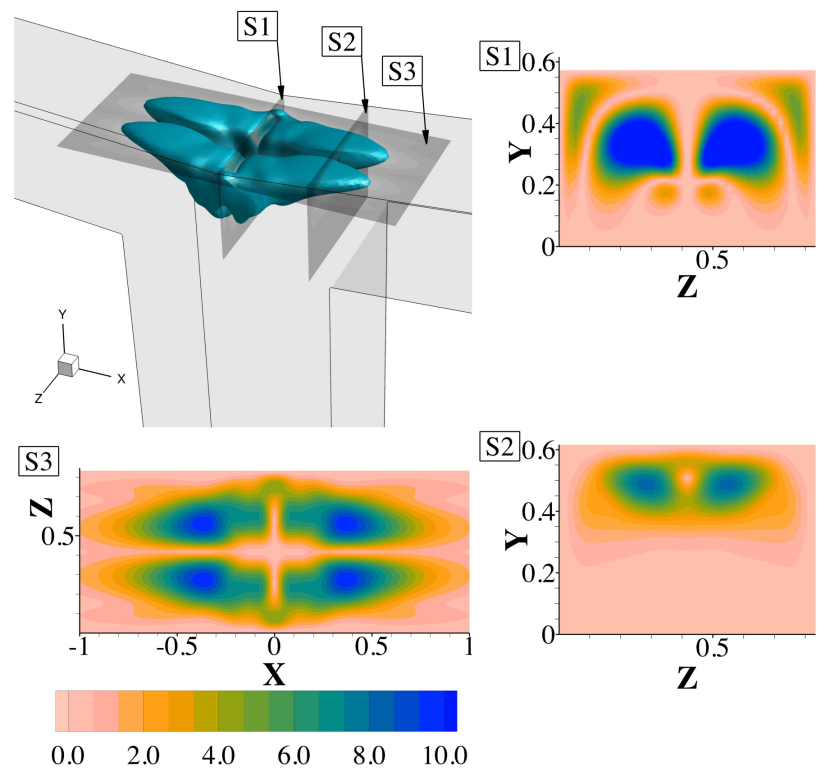

Figure 9: Isosurface of the scalar sensitivity field $S$ containing the core of the instability and distribution of $S$ at the three sections S1, S2 and S3 highlighted in the 3D figure for the case at $\alpha=+5^{\circ}$ and $R e=160$. 
differences related to the two geometries and, thus, to the angle $\alpha$ in the considered range, is mainly in the position of the core in the $y$-direction. In particular, as also discussed in Ref. [8] for a T-mixer, the instability core is located in the recirculation regions at the confluence of the two inlet streams. These regions vary with the geometry, as discussed in Sec. 3, and in particular recirculations are progressively longer and more distant from the top wall (in the $y$-direction) of the mixer as the angle $\alpha$ is decreased. Thus, the same effect can be observed for the instability core. In order to show how the field $S$ is distributed inside the instability core, a few sections are also reported in Figs. 8 and 9. Sections S1 and S2 (see referenced figures) show that the core is concentrated in two lobes which are symmetrically placed with respect to the $z$-axis, the intensity being maximum on the symmetry plane and decreasing with the distance from it. The distance of the core from the plane $y=0$, which is a common plane as $\alpha$ is varied, is slightly different between $\alpha=-20^{\circ}$ and $\alpha=+5^{\circ}$, as well as the distribution of $\mathrm{S}$ inside the core. Moreover, the intensity of $\mathrm{S}$ is generally larger for $\alpha=+5^{\circ}$, indicating a larger sensitivity of the eigenvalue to a structural perturbation of the linearized stability equations. This fact can also be caused by the differences in terms of Reynolds number between Fig. 8 and Fig. 9. Both values of $R e$ are slightly sub-critical, thus they are equivalent in comparison with $R e_{c}$, but they differ quantitatively, $R e$ being 100 and 160 for $\alpha=-20^{\circ}$ and $\alpha=+5^{\circ}$, respectively. It is shown in the literature that sensitivities generally increase with $R e$ (see, for instance, [22]), thus this difference in amplitude, whose maximum value is of the order of $35 \%$, can be influenced by the differences in $R e$. The largest qualitative differences in the distribu- 
tion of $S$ between $\alpha=-20^{\circ}$ and $\alpha=+5^{\circ}$ can be observed in slice S3 reported in Fig. 8 and Fig. 9. The distribution of $S$, which of course is influenced by the position of the considered section, respects the same symmetries of the geometry. However, for $\alpha=+5^{\circ}$ it is localized in 4 main lobes, while the distribution is articulated in 8 smaller lobes for $\alpha=-20^{\circ}$. The connection between this distribution and the local flow topology is not completely clear, although it is known that the peaks in sensitivity are usually correlated with recirculations.

\section{Concluding Remarks}

The flow in micromixers having different geometries has been investigated by numerical simulation and stability analysis, with emphasis on the onset of the engulfment regime. This regime is important in micromixers because it leads to a significant increase of the mixing between the incoming flows. Thus, it is of interest to anticipate it in terms of flow Reynolds number so as to obtain efficient mixing at the low velocities typical of micromixers. To this purpose we have considered mixers that can be obtained from a $\mathrm{T}$ configuration by varying the angle between the axis of the inflow channels and that of the outflow conduit. The complementary of this angle, $\alpha$, is varied between $\alpha=-20^{\circ}$ (arrow-like mixers) and $\alpha=+5^{\circ}$ (Y-mixers). Numeri-

cal simulations show that the engulfment regime is anticipated in terms of critical Reynolds number $R e_{c}$ as $\alpha$ is decreased, and this explains why more attention has been paid in the present paper to arrow-like mixers. Despite the differences in terms of $R e_{c}$, the engulfment regime and the resulting flow topology is moderately affected by the geometry variations, the main differ- 
ences being confined at the confluence region between the incoming flows.

Further insight into the engulfment regime is obtained by applying stability analysis to the flow. This analysis allows the identification of the supercritical pitchfork bifurcation leading to the engulfment regime and to provide a more refined estimation of $R e_{c}$. Moreover, an adjoint-based structural sensitivity analysis has been carried out to identify the core of the instability. As for the flow field, also the stability modes and core are moderately affected by the geometry changes. However, it is shown that the differences observed in the flow field for varying $\alpha$, although confined in a small zone, are indeed located inside the core of the instability, i.e. the space region where the instability leading to the engulfment onset originates. This explains the significant effects of even small variations of $\alpha$ on the critical Reynolds number value.

As a final practical remark, the results of the present paper show that even modest geometrical changes which can be easily implemented in real devices, as those considered here, can have a large effect for the onset of the engulfment regime and, consequently, for the mixing efficiency at the low velocities which are typical for micro devices. In the present study, the geometrical modifications are obtained by varying the direction of the inlet channel axis; more generally, the sensitivity analysis indicates that effective modifications can be all those that influences the recirculation regions near the mixer top walls at the confluence of incoming streams. 


\section{Acknowledgments}

The authors wish to thank CINECA computing center (Bologna, Italy) for allowance of computational resources on FERMI supercomputer under the ISCRA programme.

\section{References}

[1] V. Kumar, M. Paraschivoiu, K. Nigam, Single-phase fluid flow and mixing in microchannels, Chemical Engineering Science 66 (2011) 13291373.

[2] M. Engler, N. Kockmann, T. Kiefer, P. Woias, Numerical and experimental investigations on liquid mixing in static micromixers, Chemical Engineering Journal 101 (2004) 315-322.

[3] M. Hoffmann, M. Schlüter, N. Räbiger, Experimental investigation of liquid-liquid mixing in T-shaped micro-mixers using $\mu$-LIF and $\mu$-PIV, Chemical Engineering Science 61 (2006) 2968-2976.

[4] D. Bothe, C. Stemich, H. Warnecke, Fluid mixing in a T-shaped micromixer, Chemical Engineering Science 61 (2006) 2950-2958.

[5] S. Dreher, N. Kockmann, P. Woias, Characterization of laminar transient flow regimes and mixing in T-shaped micromixers, Heat Transfer Engineering 30 (2009) 91-100.

[6] S. Thomas, T. Ameel, J. Guilkey, Mixing kinematics of moderate Reynolds number flows in a T-channel, Physics of Fluids 22 (2010) 013601. 
[7] N. Kockmann, D. Roberge, Transitional Flow and Related Transport Phenomena in Curved Microchannels, Heat Transfer Engineering 32 (2011) 595-608.

[8] A. Fani, S. Camarri, M.V. Salvetti, Investigation of the steady engulfment regime in a three-dimensional T-mixer, Physics of Fluids 25 (2013) 064102.

[9] A. Fani, S. Camarri, M.V. Salvetti, Unsteady asymmetric engulfment regime in a T-mixer, Physics of Fluids 26 (2014) 074101.

[10] A. Soleymani, H. Yousefi, I. Turunen, Dimensionless number for identification of flow patterns inside a T-micromixer, Chemical Engineering Science 63 (2008) 5291-5297.

[11] S. Cherlo, S. Pushpavanam, Effect of depth on onset of engulfment in rectangular micro-channels, Chemical Engineering Science 65 (2010) 6486-6490.

[12] H. Yousefi, W. Ratchananusorn, I. Turunen, A. Soleymani, Pressure drop in micro T-mixers, Journal of Micromechanics and Microengineering 20 (2010) 15029-15035.

[13] C. Galletti, M. Roudgar, E. Brunazzi, R. Mauri, Effect of inlet conditions on the engulfment pattern in a T-shaped micro-mixer, Chemical Engineering Journal 185 (2012) 300-313.

[14] R. Poole, A. M, G. AP, Bifurcation in a T-channel junction: Effects of aspect ratio and shear-thinning, Chemical Engineering Sciences 104 (2013) 839-848. 
[15] T. Andreussi, G. Galletti, R. Mauri, S. Camarri, M.V. Salvetti, Flow regimes in T-shaped micro-mixers, under review in Computers \& Chemical Engineering (2015).

[16] P. Chatwin, P. Sullivan, The effect of aspect ratio on the longitudinal diffusivity in rectangular channels, Journal of Fluid Mechanics 120 (1982) 347-358.

[17] J. Happel, H. Brenner, Low Reynolds Number Hydrodynamics, Prentice Hall, 1965.

[18] O. Marquet, D. Sipp, L. Jacquin, Sensitivity analysis and passive control of cylinder flow, Journal of Fluid Mechanics 615 (2008) 221-252.

[19] J. W. L. Paul F. Fischer, S. G. Kerkemeier, NEK5000 Web page, 2008. Http://nek5000.mcs.anl.gov.

[20] J. Jeong, F. Hussain, On the identification of a vortex, Journal of Fluid Mechanics 285 (1995) 69-94.

[21] F. Giannetti, P. Luchini, Structural sensitivity of the first instability of the cylinder wake, Journal of Fluid Mechanics 581 (2007) 167-197.

[22] S. Camarri, B.E.G. Fallenius, J.H.M. Fransson, Stability analysis of experimental flow fields behind a porous cylinder for the investigation of the large-scale wake vortices, Journal of Fluid Mechanics 715 (2013) 499-536. 